\title{
Atomic Scale Study of Lomer-Cottrell and Hirth Lock Dislocations in CdTe
}

\author{
Tadas Paulauskas ${ }^{1}$, Chris Buurma ${ }^{1}$, Brian Stafford ${ }^{1}$, Cyrus Sun ${ }^{2}$, Maria Chan ${ }^{3}$, Sivananthan \\ Sivalingham ${ }^{1}$, Moon $\mathrm{Kim}^{2}$ and Robert F. Klie ${ }^{1}$ \\ 1. University of Illinois at Chicago, Department of Physics, Chicago, USA \\ 2. University of Texas at Dallas, Department of Materials Science and Engineering, Dallas, USA \\ 3. Argonne National Laboratory, Center for Nanoscale Materials, Argonne, USA
}

Many useful and also detrimental properties of solids can be traced back to the underlying structure of dislocations and their behavior. Their presence has been studied extensively studied in the context of crystal growth on lattice mismatched substrates and dislocations may significantly alter a material's mechanical, thermal and opto-electronic properties [1]. Experimental knowledge detailing atomically resolved chemical structure of dislocation cores is highly desirable to advance fundamental understanding of these ubiquitous structures. In this study we present atomic scale analysis of two wellknown dislocations junctions in CdTe, the Lomer-Cottrell (L-C) and the Hirth lock. Despite being textbook examples of the lowest elastic energy stair-rod dislocations their atomic structure, in particularly in zinc-blende CdTe, were unknown. Stair-rod dislocations, usually identified in terms of their lack of mobility, may have important effects on the mechanical properties which raise concerns given the trend of continuous miniaturization of semiconductor devices. Furthermore, being pure-edge dislocations, L-C and Hirth locks are likely to be found in low-angle tilt grain boundaries.

The dislocations under investigation here were studied as occurring naturally in poly-crystalline CdTe thin films incorporated into traditional $\mathrm{CdS} / \mathrm{CdTe}$ solar cells. The post-deposition $\mathrm{CdCl}_{2}$ annealing process, commonly used in commercial poly-CdTe-based solar cells, was not performed on these samples in order to investigate the dislocations without any extrinsic dopants. Characterization is carried out in the aberration-corrected cold-field emission JEOL JEM-ARM200CF scanning transmission electron microscope (STEM) using high-angle annular dark field (HAADF). Chemical composition of the dislocation cores is demonstrated via atomic-column-resolved X-ray energy dispersive spectroscopy (XEDS) using windowless silicon drift detector, the Oxford Instruments X-Max 100TLE. Local strain fields associated with dislocations are measured from the images via the Geometrical Phase Analysis (GPA).

In order to confirm identity of the dislocations we constructed Burgers circuits specially tailored for dislocation junctions since faulted material cannot be crossed twice in a circuit. To explain reaction steps and core configurations of the resulting dislocation junctions a double Thompson's tetrahedron is used which can take into account intrinsic/extrinsic stacking faults and polar variants of dislocations in CdTe [2].

Figure 1 a) shows X-ray spectrum map of L-C dislocation polar core variant which is seen to be composed of three Cd atomic columns and one Te at the center. In b) the Burgers circuit construction is used to identify nature of the dislocation as indicated. An unfaulted gap in the material (dashed box) is used to pass the circuit through. Partial dislocation introduced in this way is taken into account by the second small circuit. Figure 2 a) shows the Hirth lock dislocation. The core is more compact and is composed of two atomic columns (Te and Cd). Figures $2 \mathrm{~b}$ ) and c) indicate the dislocation reaction steps in terms of the partial dislocations on two different $\{111\}$ planes, which result in formation of the core. 


\section{References:}

[1] Sutton, A. P. \& Balluffi, R. W., "Interfaces in Crystalline Materials”, New York: Oxford Science Publications, (1995).

[2] Paulauskas, T. et.al.,” Atomic scale study of polar Lomer-Cottrell and Hirth lock dislocation cores in CdTe”, Acta Cryst. - A, (2014).

[3] This research is supported by a grant from the Department of Energy Sunshot Program (DOE DEEE0005956).
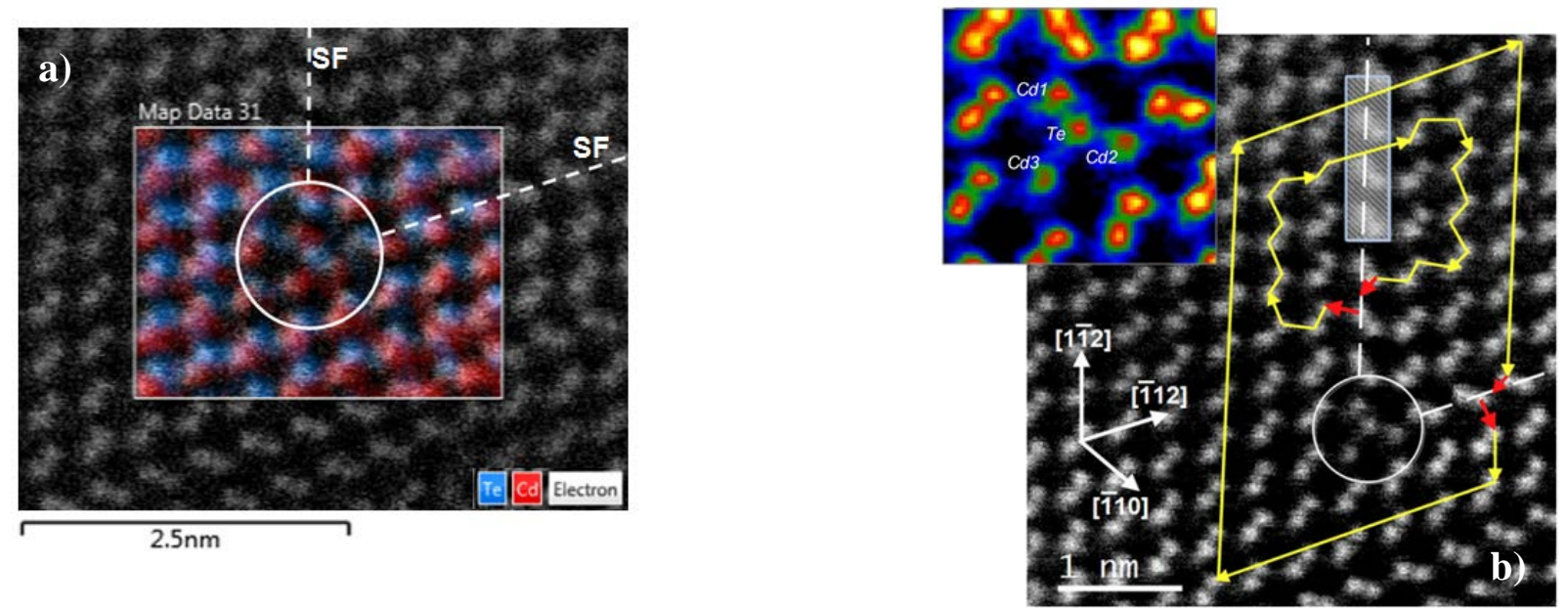

Figure 1. a) X-ray spectrum image of the L-C dislocation core (circled) which is associated with two intrinsic stacking faults (dashed SF). b) Two Burgers circuits construction is shown on an HAADF image for determination of Burgers vector of the stair-rod dislocation. Relevant crystallographic directions are shown as well. Inset shows magnified view of the core.
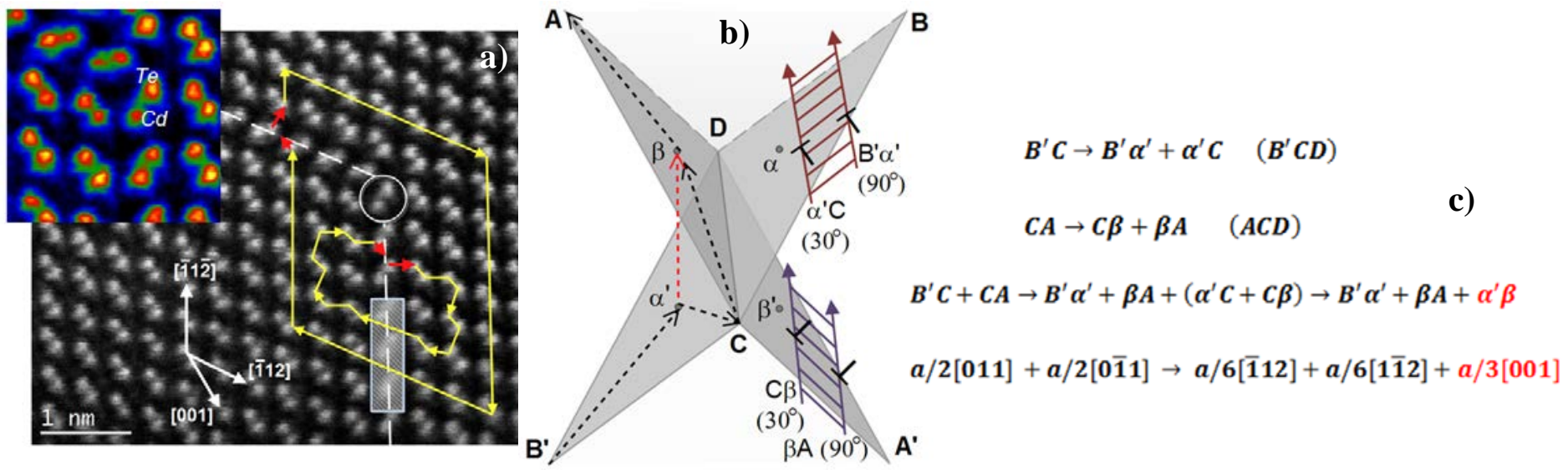

Figure 2. a) HAADF image and Burgers circuits for the Hirth lock. b) Double Thompson's tetrahedron for the Hirth lock, showing dissociated perfect dislocations involved in the reaction on $\{111\}$ planes. c) Dislocation reaction steps in terms of partial dislocations Burgers vectors, as per figure b). 